\title{
El análisis de las desigualdades en salud: una breve revisión de los métodos
}

\author{
Andrew M. Jones \\ Department of Economics and Related Studies, University of York \\ Cristina Hernández Quevedo \\ European Observatory of Health Systems and Policies, \\ London School of Economics and Political Science, Reino Unido \\ Nigel Rice \\ Centre for Health Economics, University of York, Reino Unido \\ Ángel López Nicolás
Departamento de Economía, Universidad Politécnica de Cartagena y CRES, España
}

\section{Resumen}

La reducción de las desigualdades en salud es uno de los objetivos más comunes en los sistemas de salud a nivel mundial. Los economistas han respondido a este desafio desarrollando métodos empíricos que proporcionan instrumentos útiles para cuantificar el grado de desigualdad en la distribución de medidas de salud, comparar las desigualdades en salud en el espacio y en el tiempo, identificar los factores que causan las desigualdades en salud y simular los efectos de diferentes políticas en dichas desigualdades. Este artículo ofrece una revisión concisa de los métodos más recientes y relevantes en esta área de investigación de rápido crecimiento, indicándole al lector tanto trabajo seminal como interesantes aplicaciones empíricas.

Palabras clave: Desigualdades en salud, microdatos.

Clasificación JEL: I12, C23

\begin{abstract}
The reduction of health inequalities is one of the most common policy aims of health systems worldwide. Economists have responded to this challenge by developing empirical methods that provide useful ways to quantify the degree of inequality in the distribution of measures of health, to compare health inequalities across space and time, to identify the factors that drive health inequalities and to simulate the effects on health inequalities of alternative policy scenarios. This paper offers a concise review of the most recent and relevant methods in this fast growing area of research, pointing the reader to both seminal work and interesting empirical applications
\end{abstract}

Key words: Health inequalities, micro data.

JEL Classification: I12, C23

Se agradece el apoyo recibido por parte del Ministerio de Educación y Ciencia a través del proyecto SEJ2005-08783-C04 02/ECON, que ha sido cofinanciado con FEDER. 


\section{Introducción}

La reducción de las desigualdades en salud es uno de los objetivos más comunes en los sistemas de salud a nivel mundial. Los economistas han respondido a este desafío desarrollando métodos empíricos que proporcionan instrumentos útiles para cuantificar el grado de desigualdad en la distribución de medidas de salud, comparar las desigualdades en salud en el espacio y en el tiempo, identificar los factores que causan las desigualdades en salud y simular los efectos de diferentes políticas en dichas desigualdades. Este artículo ofrece una revisión concisa de los métodos más recientes y relevantes en esta área de investigación de rápido crecimiento, indicándole al lector tanto trabajo seminal como interesantes aplicaciones empíricas.

La sección 2 está dedicada a la exposición de los métodos basados en la curva de concentración e índices de concentración, que han sido utilizados frecuentemente en el análisis de las desigualdades socioeconómicas en salud. La sección 3 describe cómo los modelos econométricos de salud pueden ser explotados fructíferamente para descomponer la desigualdad en salud en diferentes factores explicativos. La sección 4 revisa los desarrollos recientes en la extensión del análisis de la desigualdad a un contexto longitudinal. La sección 5 concluye y destaca algunas aplicaciones para el caso español.

\section{Métodos basados en las curvas de concentración}

En la economía de la salud, los métodos basados en las curvas e índices de concentración han sido utilizados para medir desigualdades e inequidades (Wagstaff y van Doorslaer, 2000). La curva de concentración (CC) y el índice de concentración (CI) de salud proporcionan medidas de desigualdad relativa en salud relacionada con la renta (Wagstaff, Van Doorslaer y Paci, 1989). Wagstaff, Paci y van Doorslaer (1991) revisan y comparan las propiedades de las curvas e índices de concentración con medidas alternativas de desigualdad en salud. Dichos autores argumentan que las principales ventajas de éstos son las siguientes: capturan la dimensión socioeconómica de las desigualdades en salud; utilizan información de toda la distribución frente al uso de los extremos de la distribución únicamente; ofrecen la posibilidad de ser representados visualmente a través de la curva de concentración y permiten la comprobación de relaciones de dominancia.

El índice de concentración se deriva de la curva de concentración. Esto se ilustra en la Figura 1 para una medida de enfermedad. La muestra de interés está ordenada según su situación socioeconómica. De tal forma que, si la renta es utilizada como la variable relevante para ordenar a los individuos, el eje horizontal comienza con el individuo más pobre en la sociedad y progresa a través de la distribución de la renta hasta el individuo más rico en dicha sociedad. Este ranking relativo según el nivel de renta se representa gráficamente frente a la proporción acumulada de enfermedad en el eje vertical. Esto asume que existe una medida cardinal de enfermedad, que puede 
FIGURA 1

CURVA DE CONCENTRACIÓN PARA MALA SALUD

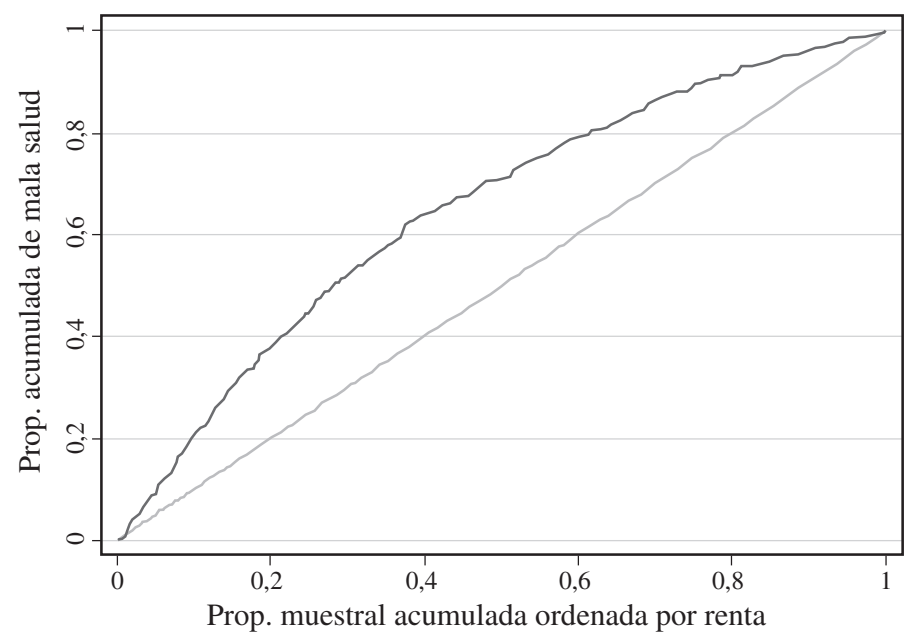

ser comparada y agregada entre individuos. La línea de 45 grados muestra la línea de igualdad perfecta, a lo largo de la cual las proporciones de enfermedad de la población son proporcionales a la renta, de tal forma que el $20 \%$ de los individuos más pobres experimentan un $20 \%$ del total de enfermedad en la población, y así sucesivamente. En realidad, es improbable que la igualdad perfecta exista en la distribución de enfermedad. La desigualdad concentrada en los más pobres se ilustra con la curva cóncava en la figura - la curva de concentración. En el ejemplo mostrado, el 20\% de individuos con menor nivel de renta experimentan más del 20\% de las enfermedades. El hecho de que la curva de concentración esté situada por encima de la línea de igualdad perfecta indica que hay desigualdad en enfermedad concentrada en los más pobres. El tamaño de la desigualdad puede ser resumido por el índice de concentración de salud, que corresponde a dos veces el área entre la curva de concentración y la línea de 45 grados.

Hay varias formas de expresar el índice de concentración (C) algebraicamente. Una forma conveniente es:

$$
C=\frac{2}{\mu} \sum_{i=1}^{N}\left(y_{i}-\mu\right)\left(R_{i}-\frac{1}{2}\right)=\frac{2}{\mu} \operatorname{cov}\left(y_{i}, R_{i}\right)
$$

Esta expresión muestra que el valor del índice de concentración es igual a la covarianza entre salud individual (y) y el ranking relativo del individuo (R), escalado por la media de salud en la población ( $\mu$ ).La expresión se multiplica por 2 para asegurar que el índice de concentración toma valores entre -1 y +1 . Escribiendo el índice de concentración de esta forma se enfatiza que es un indicador del grado de asociación entre el nivel de salud del individuo y su posición relativa en la distribución de la renta. 
La desigualdad socioeconómica en salud es citada extensamente como preocupación por los policymakers en el ámbito de la salud, sin embargo este interés podría ir más allá. Podrían también estar preocupados por otras fuentes de desigualdad que son capturadas a través de una medida de desigualdad en salud total. Esto se puede analizar utilizando las curvas de Lorenz de salud y la desigualdad puede ser medida usando el coeficiente de Gini para la desigualdad en salud (Le Grand, 1989; Wagstaff, Paci y van Doorslaer, 1991). El atractivo de este enfoque es la existencia de una relación directa entre el índice concentración y el coeficiente de Gini para salud: el índice de concentración es proporcional al coeficiente de Gini, donde el factor de proporcionalidad viene dado por el ratio entre el coeficiente de correlación para salud y el ranking según el nivel de renta, y la correlación de coeficientes entre salud y ranking según el nivel de salud (Kakwani, 1980; van Doorslaer y Jones, 2003). Esto significa que es fácil moverse entre estas medidas particulares de desigualdad socioeconómica en salud y desigualdad pura en salud.

La literatura sobre la desigualdad en renta hace una distinción entre órdenes parciales, basados en las curvas de Lorenz o de concentración, y órdenes completos, basados en números índices tales como los índices de Gini y de concentración. Un orden parcial significa que algunas combinaciones de distribuciones pueden ser ordenadas sin ambigüedad, pero no todas. La ambigüedad surge si las curvas de Lorenz o de concentración para dos distribuciones se cruzan entre sí. Para obtener un orden completo de las distribuciones, los coeficientes de Gini e índices de concentración incorporan juicios normativos concretos sobre el peso dado a los individuos en diferentes puntos de la distribución de la renta y, por tanto, incluyen un grado concreto de aversión a la desigualdad. La sensibilidad de los resultados a la aversión a la desigualdad puede ser evaluada utilizando los índices de concentración extendidos (Wagstaff, 2002). Éstos añaden un parámetro adicional que puede variar desde la neutralidad en la desigualdad (sin preocupación por la desigualdad) hasta la aversión extrema a la desigualdad (lexi-min rawlsiano).

Los índices de Gini y de concentración son medidas de la desigualdad relativa y no tienen en cuenta la compensación entre equidad y eficiencia. Esta compensación puede ser capturada por las curvas de concentración o de Lorenz generalizadas, que multiplican la curva de concentración o de Lorenz por el nivel medio de enfermedad. Estas curvas permiten comparaciones entre países y proporcionan evidencia sobre relaciones de dominancia generalizada. La altura vertical de la curva, en el eje derecho, muestra el nivel medio de enfermedad y la curvatura refleja las desigualdades en enfermedad relacionadas con la renta. Si la curva correspondiente a un país se sitúa por encima de la curva de otro país en todo momento, esto significa que este país tiene una mayor prevalencia de enfermedad total y un mayor grado de desigualdades relacionadas con la renta en su distribución. El hecho de que la curva para un país domine a la de otro país proporciona un orden sin ambigüedad. En la práctica, el orden de los países podría no estar claro ya que las curvas se cruzarían entre sí con frecuencia, obteniéndose únicamente un orden parcial. 
Un orden completo puede ser impuesto utilizando el índice de concentración generalizado, $\mu(1-\mathrm{CI})$, el cual proporciona un índice único que captura la compensación entre la media de la distribución ( $\mu$ ) y el nivel de desigualdad (CI). Este índice puede ser interpretado como una forma simple de función de bienestar social. Para añadir mayor flexibilidad y relajar las premisas sobre el grado de aversión al riesgo que lleva implícita la medida estándar del índice de concentración, este enfoque puede ser combinado con diferentes grados de aversión a la desigualdad, a través del índice de concentración extendido. Esto proporciona un índice de logro de salud citado por Wagstaff (2002), que resume la compensación entre equidad y eficiencia para diferentes grados de aversión a la desigualdad. Diferentes valores de aversión a la desigualdad ( $v)$ permiten diferencias en el peso que se le da a los individuos más pobres al calcular el índice de concentración. Por tanto, el índice de logro viene dado por la siguiente expresión:

$$
I A(v)=\mu(1-C(v))=\frac{1}{n} \sum_{i=1}^{n} y_{i} v\left(1-R_{1}\right)^{(v-1)}
$$

donde $y_{i}$ es la medida de salud o enfermedad para el individuo i y $R_{i}$ es su orden relativo en la distribución de la renta. Cuando $v$ toma valor 1 , el índice de logro se simplifica siendo igual al nivel medio de enfermedad ( $\mu$ ); si toma valor 2 , la fórmula se basa en el índice de concentración estándar; valores mayores de $v$ implican mayor peso para la preocupación por la equidad. Este índice ha sido aplicado a datos del Panel de Hogares Comunitario (PHOGUE) en Hernández Quevedo et al. (2006).

\section{Análisis de descomposición}

El índice de concentración tiene el atractivo de poder ser descompuesto en factores (Rao, 1969; Kakwani, 1980), como es el caso del coeficiente de Gini de desigualdad en renta. Por ejemplo, esta propiedad ha sido utilizada en el pasado para descomponer el índice de concentración para la financiación de la atención sanitaria en diferentes fuentes de pagos de la atención sanitaria tales como impuestos, contribuciones a la seguridad social, cargos al usuario, etcétera. Un artículo reciente de Wagstaff, van Doorslaer y Watanabe (2003) explota el resultado siguiente: si una ecuación de demanda de salud en forma reducida es separable aditivamente,

$$
y_{i}=\alpha+\sum_{k} \beta_{k} x_{k i}+\varepsilon_{i}
$$

entonces, como el índice de concentración se puede descomponer aditivamente —lo cual proviene del hecho de que la covarianza de una combinación lineal es igual a la combinación lineal de las covarianzas - el índice de concentración total para salud puede ser escrito como sigue,

$$
C=\sum_{k}\left(\beta_{k} \bar{x}_{k} / \mu\right) C_{k}+G C_{\varepsilon} / \mu=C_{\hat{y}}+G C_{\varepsilon} / \mu
$$


Éste tiene la forma conveniente por la que $\mathrm{C}$ puede ser separado en dos partes: el primer término puede ser considerado como el componente explicado $\left(\mathrm{C}_{\dot{y}}\right)$ y el segundo término como el componente no explicado. Dentro del componente explicado, hay una contribución para cada uno de los regresores $(x)$ y esto viene dado por el producto de dos términos. El primer término es la elasticidad de salud con respecto a aquella variable, por ejemplo la elasticidad-renta de salud, y el segundo término es el índice de concentración de esa variable, por ejemplo, en el caso de la renta éste sería el coeficiente de Gini.

Más interesante que una mera descomposición de la desigualdad país a país es la descomposición de las diferencias en las desigualdades en salud entre países en, por un lado, las diferencias en desigualdad en los determinantes de salud, y por otra parte, las diferencias en los efectos de salud de estos determinantes entre países. Esto no es directo, porque algunas diferencias (por ejemplo, las diferencias en la media de $x_{k}$ ) podrían ser compensadas por otras diferencias (por ejemplo, diferencias en el grado de desigualdad en $x_{k}$ ). Además, estos cambios en general no serán independientes entre sí y el método de descomposición tiene que tener en cuenta estas interdependencias. Un enfoque para solucionar este problema es aplicar un método de descomposición Oaxaca. Si denotamos como $\eta_{k i}$ la elasticidad de $y$ respecto a $x_{k i}$ para el país $i$, y por $C_{k i}$ el índice de concentración del determinante $k$ en el país $i$, entonces podemos aplicar el método Oaxaca eligiendo el país $i$ para los valores de referencia de $\eta_{k i}$ y el país $j$ para los valores de $C_{k i}$ y así obtener:

$$
\Delta C=C_{i}-C_{j}=\sum_{k} \eta_{k i}\left(C_{k i}-C_{k j}\right)+\sum_{k} C_{k j}\left(\eta_{k i}-\eta_{k j}\right)
$$

siendo la alternativa:

$$
\Delta C=C_{i}-C_{j}=\sum_{k} \eta_{k j}\left(C_{k i}-C_{k j}\right)+\sum_{k} C_{k i}\left(\eta_{k i}-\eta_{k j}\right)
$$

Entonces $\Delta C_{k}$, la contribución de cualquier variable $x_{k}$ a $\Delta C$, es igual a la suma de dos términos, i.e.:

$$
\Delta C_{k}=\eta_{k i}\left(C_{k i}-C_{k j}\right)+C_{k j}\left(\eta_{k i}-\eta_{k j}\right)
$$

expresados en términos del producto de una elasticidad y un índice de concentración, que no presenta unidades naturales. Es necesario tener en cuenta que, mientras las ecuaciones producen idénticos $\Delta C_{k}$ 's y $\Delta C_{k}$ 's, su descomposición no es única y podría llevar a resultados que difieren levemente dependiendo de la elección de $i$ o $j$ como el «país índice» o país de referencia para los estimadores de $\eta_{k i}$ y $C_{k i}$.

\section{Medidas para datos longitudinales}

Los datos longitudinales ofrecen información sobre la dinámica de la salud y la renta de los individuos y su impacto en la desigualdad sobre los períodos, extendién- 
dose más que la encuesta de sección cruzada para un año concreto. Si la política sanitaria - y la política social en general — está preocupada por el historial de vida (ver p.e. el argumento conocido como «fair innings» descrito por Williams y Cookson, 2000), entonces la perspectiva a largo plazo proporcionada por los datos de panel puede producir información adicional útil. Recientemente, basándose en la literatura sobre la desigualdad en renta, Jones y López Nicolás (2004) han explorado la información adicional que puede ser obtenida al utilizar datos de panel. Los trabajos sobre movilidad en renta se han centrado en comparar la distribución de la renta utilizando dos perspectivas: en primer lugar, una perspectiva de corto plazo o de sección cruzada y en segundo lugar, una perspectiva a largo plazo donde la renta es agregada sobre una serie de períodos. Si el ranking del individuo según la renta difiere entre el corto y largo plazo, hay evidencia de movilidad en renta. Una forma de medir este fenómeno es a través del índice de movilidad en renta propuesto por Shorrocks (1978).

Jones y López Nicolás (2004) aplican los principios utilizados por Shorrocks (1978) a las desigualdades en salud relacionadas con la renta. Los autores muestran que las desigualdades en salud relacionadas con la renta pueden ser o bien mayores o menores en el largo plazo que en el corto plazo y que, una vez más, estos cambios pueden ser medidos a través de un índice de la movilidad en renta relacionada con la salud, basado en el índice de concentración. Es útil medir cuánto la perspectiva longitudinal, donde $\mathrm{N}$ individuos son observados durante $\mathrm{T}$ períodos, altera el resultado que podría aparecer de una serie de secciones cruzadas. Jones y López Nicolás (2004) definen un índice de movilidad en renta relacionada con salud para medir la diferencia entre desigualdad a largo plazo y a corto plazo:

$$
\begin{aligned}
& M^{T}=1-\frac{C I^{T}}{\sum_{\substack{t \\
t}} w_{t} C I^{t}}=\frac{2}{N \sum_{\substack{t \\
t}} \bar{y}^{t} C I^{t}}\left(\sum_{i} \sum_{t}\left(y_{i t}-\bar{y}_{t}\right)\left(R_{i}^{t}-R_{i}^{T}\right)\right) \\
& w_{t}=\frac{\bar{y}^{t}}{T \overline{\bar{y}}^{T}} \\
& y \\
& \text { (i) } \bar{y}^{t}=\frac{\sum_{i} y_{i t}}{N} \\
& i=1, \ldots, N ; \mathrm{t}=1, \ldots, \mathrm{T} \\
& \text { (ii) } \overline{\bar{y}}^{T}=\frac{\sum_{t} \sum_{i} y_{i t}}{N T}=\frac{\sum_{t} \bar{y}^{t}}{T}
\end{aligned}
$$

donde:

$\mathrm{y}_{\mathrm{it}}$ : una medida cardinal de enfermedad para el individuo $\mathrm{i}(\mathrm{i}=1, \ldots, \mathrm{N})$ en el momento $t(t=1, \ldots, T)$.

$\mathrm{y}_{\mathrm{i}}^{\mathrm{T}}=(1 / \mathrm{T})_{\mathrm{t}} \mathrm{y}_{\mathrm{it}}$ : la media para el individuo i después de $\mathrm{T}$ períodos. 


\section{$\mathrm{R}_{\mathrm{i}}^{\mathrm{t}}$ : $\quad$ orden relativo del individuo $\mathrm{i}$ en la distribución de $\mathrm{N}$ rentas en el período $t$. \\ $\mathrm{R}_{\mathrm{i}}^{\mathrm{T}}$ : $\quad$ orden relativo del individuo i en la distribución de $\mathrm{N}$ rentas me- dias después de $\mathrm{T}$ períodos.}

Esta definición muestra que el índice de movilidad en renta relacionada con salud es «uno menos el ratio por el cual el índice de concentración para la distribución conjunta de las medias longitudinales difiere de la media ponderada de los índices de concentración de las distintas secciones cruzadas, debido a la asociación sistemática entre salud y cambios en el orden del individuo según la renta» (Jones y López Nicolás, 2004). Cuanto mayor sea la discrepancia entre las medidas de desigualdad a corto y largo plazo, mayor es el valor de $\mathrm{M}^{\mathrm{T}}$. La ausencia de discrepancia implica que $\mathrm{M}^{\mathrm{T}}$ es igual a cero. El signo del índice viene dado por la covarianza en el segundo término de la expresión (8). Esto es, un valor negativo del índice implica que las desigualdades a largo plazo son mayores que el promedio de las desigualdades en cada subperíodo, y viceversa.

Jones y López Nicolás (2004) muestran que el índice de concentración a largo plazo para la media de salud en $\mathrm{T}$ períodos (denotado $\mathrm{CI}^{\mathrm{T}}$ ) es la suma de dos términos. El primer término es una suma ponderada de índices de concentración a corto plazo (esto es, el índice de concentración para cada una de las oleadas denotados como $\mathrm{CI}^{\mathrm{t}}$ ), mientras que el segundo término refleja la covarianza entre niveles de salud y fluctuaciones en el orden proporcionado por la renta a lo largo del tiempo. Si el ranking según la renta se mantiene constante a lo largo del tiempo, un resultado estándar de descomposición para los índices de concentración implica que el índice de concentración para la media a lo largo del tiempo es igual a la media (ponderada) de los índices de concentración. Sin embargo, el ranking del individuo según la renta podría cambiar a lo largo del tiempo y podría ser el caso que, por ejemplo, los individuos que se desplazan hacia posiciones inferiores en el ranking según la renta, tengan peor salud que la media. El efecto de tales relaciones no puede ser detectado con datos de sección cruzada. Si los individuos cambian su posición a lo largo de los T períodos, y estos cambios están sistemáticamente relacionados con la salud, entonces el segundo término en la descomposición no será igual a cero. Si es positivo, entonces aquellos individuos que se mueven hacia posiciones superiores en el ranking según la renta en el sentido que su orden en la distribución de la renta en el largo plazo es superior que su ranking cuando la renta está medida en un corto período de tiempo- disfrutan un nivel de enfermedad inferior a la media. Por tanto, esto significa que aquellos individuos que se mueven hacia posiciones inferiores en la distribución de la renta tenderían a sufrir un nivel de enfermedad superior a la media. En este contexto, la desigualdad en salud relacionada con la renta a largo plazo sería mayor que el promedio de las medidas a corto plazo. 


\section{Conclusión}

Este artículo ha ofrecido una revisión concisa de los métodos empíricos más relevantes desarrollados recientemente por economistas para el análisis de las desigualdades en salud y su relación con la situación socioeconómica. El número de aplicaciones para el caso de España se ha incrementado en los últimos años. Por ejemplo, García Gómez y López Nicolás (2007a) han medido el grado de desigualdades en salud relacionadas con la renta en las regiones españolas, mostrando que el factor explicativo más relevante no es la desigualdad en la distribución de la renta sino la elasticidad-renta de la salud. Las razones por las que esta elasticidad es elevada en algunas regiones y prácticamente cero en otras supone un desafío tanto para los investigadores como policymakers. Otra aplicación para el caso español por García Gómez y López Nicolás (2007b) se centra en el impacto del seguro de salud privado en el grado de equidad en la utilización de la atención sanitaria, mostrando que la contribución del seguro de salud privado a la inequidad ha aumentado durante el período 1987-2001 y, por tanto, surgen interrogantes sobre la pertinencia de las deducciones en el impuesto de sociedades por contratación de planes privados de salud. En este volumen de Cuadernos Económicos del ICE, hay dos artículos que aplican los métodos anteriormente descritos: Stoyanova et al. (2008) y Costa-Font y Gil (2008) muestran cómo, disponiendo de buenos microdatos, los índices de concentración pueden ser una fuente empírica relevante para los economistas de la salud.

\section{Referencias}

[1] COSTA-FONT, J. y GIL, J. (2008). Una Primera Exploración a las Desigualdades Socioeconómicas en Morbilidad en España. En López Nicolás, A. (ed). Desigualdad, Equidad y Eficiencia en Salud y Servicios Sanitarios. Cuadernos Económicos del ICE.

[2] GARCÍA GÓMEZ, P. y LÓPEZ NICOLÁS, A. (2007a). «Public and Private Health Insurance and the Utilisation of Health Care in Spain.» Research in Economic Inequality Vol 15. pp. 169-195.

[3] GARCÍA GÓMEZ, P. y LÓPEZ NICOLÁS, A. (2007b). «Regional Differences in Socio-Economic Health Inequalities in Spain.» Documento de Trabajo 9-2007. Fundación BBVA. Bilbao. (available at http://w3.grupobbva.com/TLFB/ttfb/TLFBindex_pub.jsp)

[4] HERNÁNDEZ QUEVEDO, C., A.M. JONES, A. LÓPEZ NICOLÁS y N. RICE (2006) Socioeconomic inequalities in health: a longitudinal analysis of the European Community Household Panel, Social Science and Medicine, 63: 1246-1261.

[5] JONES, A.M. y A. LÓPEZ NICOLÁS (2004). Measurement and explanation of socioeconomic inequality in health with longitudinal data, Health Economics 13: 1015-1030.

[6] KAKWANI, N.C. (1980). Income Inequality and Poverty. Methods of Estimation and Policy Implications. Oxford University Press, Oxford.

[7] LE GRAND, J., (1989). An international comparison of ages-at-death. En J. Fox (ed.) Health inequalities in European countries. Gower, Aldershot. 
[8] RAO, V. (1969). Two decompositions of the concentration ratio. Journal of the Royal Statistical Society Series A, 132: 418-425.

[9] SHORROCKS, A. (1978). Income inequality and income mobility, Journal of Economic Theory, 19: 376-93.

[10] STOYANOVA, A., RODRÍGUEZ, M. y J. PINILLA (2008). El mapa de las desigualdades en salud por grupos de edad en España. En López Nicolás, A. (ed). Desigualdad, Equidad y Eficiencia en Salud y Servicios Sanitarios. Cuadernos Económicos del ICE.

[11] VAN DOORSLAER, E. y JONES, A.M. (2003). Inequalities in self-reported health: validation of a new approach to measurement, Journal of Health Economics, 22(1): 61-87.

[12] WAGSTAFF, A. (2002). Inequality aversion, health inequalities and health achievement. Journal of Health Economics, 21: 627-641.

[13] WAGSTAFF, A., PACI, P. y VAN DOORSLAER, E., (1991). On the measurement of inequalities in health, Social Science and Medicine, 33: 545-557.

[14] WAGSTAFF, A. y VAN DOORSLAER, E. (2000). Equity in health care finance and delivery. En Culyer, A.J. y J.P. Newhouse (eds.), Handbook of health economics. Amsterdam, Elsevier, vol 1B, 1803-1862.

[15] WAGSTAFF, A., VAN DOORSLAER, E. y PACI, P. (1989). Equity in the finance and delivery of health care: some tentative cross-country comparisons. Oxford Review of Economic Policy 5, 89-112.

[16] WAGSTAFF, A, VAN DOORSLAER, E., WATANABE, N. (2003). On decomposing the causes of health sector inequalities with an application to malnutrition inequalities in Vietnam, Journal of Econometrics, 112: 207-223.

[17] WILliAMS, A. y R. COOKSON (2000). Equity in health. En Culyer, A.J. y J.P. Newhouse (eds), Handbook of health economics. Amsterdam, Elsevier, vol 1B, 18631910 . 